\title{
Evaluating Restoration Methods A Cross A Range of Plant Communities Dominated by Invasive Annual Grasses to Native Perennial Grasses
}

\author{
Mohammed Abdulatife \\ Dubti Pastoral and Agro-pastoral Research Center, P.O.Box 16, Semera, Afar, Ethiopia
}

\begin{abstract}
The objectives of the study were to determine whether there are differences in plant community response to restoration methods \& to create awareness among the pastoralists/ agro-pastoralists on how to restore degraded rangeland. Six treatments were established: (1) ripping, mulching and seeding (2) ripping, manuling and seeding (3) ripping, mulching, manuling and seeding (4) control. The treatments were arranged in a randomized complete block design with factorial and replicated three times in each plant community. Each treatment plot was $3 \mathrm{~m}$ by $6 \mathrm{~m}$, and depending on the treatment assigned to the plot, measurements for the vegetations were taken from those plots. Seeds of perennials, large tufted and palatable grass species were selected for reseeding purpose i.e., Andropogon canaliculatus, Tetrapogon cenchriformis, Panicum coloratum and Chrysopogon plumolosus. The study was carried out over the last three rainy seasons from 2015/16 and 2017/ 2018. Data were collected from germination to dry matter yield within three years of the experimental years. Accordingly, the result showed as variation clearly observed among the restoration treatments to different plant community. There is a significant difference at $(\mathrm{P}<0.05)$ rangeland parameters collected in all Restoration methods in each seasons. A total of 18 species of grasses were identified in the study district. Of the grass species 11(61.1\%) species were perennials and 7 (38.9\%) species were annuals. In Perennial plant community with Restoration Methods of $\mathrm{Ri}+\mathrm{Ma}+\mathrm{Mu}+\mathrm{Se}$ was significant difference at $(\mathrm{P}<0.05)$ in the three consecutive seasons in Biomass production. The highest Biomass production was observed in the third season of implementation time which was 2.37 ton/ha while the lowest biomass production was observed in the first season of implementation period in control one which was 1 ton/ha. In Mixed plant community with Restoration Methods of $\mathrm{Ri}+\mathrm{Ma}+\mathrm{Mu}+\mathrm{Se}$ was significant difference at $(\mathrm{P}<0.05)$ in the three consecutive seasons in Biomass production. The highest Biomass production was observed in the third season of implementation time which was 1.75 ton/ha while the lowest biomass production was observed in the first season of implementation period in control one which was 0.83 ton/ha.

Therefore, we conclude that from this finding, perennial plant community with Restoration methods of $\mathrm{Ri}+\mathrm{Ma}+\mathrm{Mu}+\mathrm{Se}$ is more effective method to rehabilitate denuded rangeland areas in semi-arid areas and also Annual plant community with Restoration methods of $\mathrm{Ri}+\mathrm{Ma}+\mathrm{Mu}+\mathrm{Se}$ has shown dramatic result to improved range resources within short period of time. However, continuous onsite training \& practical demonstration was very crucial to enhance the adoption rate of those Restoration methods.
\end{abstract}

Keywords: Restoration Methods, plant community, Germination, Basal cover \& Biomass Yield

DOI: $10.7176 / \mathrm{JBAH} / 9-19-01$

Publication date:October $31^{\text {st }} 2019$

\section{Introduction}

The Afar Region is located in the North Eastern lowlands of Ethiopia, with the area of about $100,860 \mathrm{~km}^{2}$ (IBANRS, 2000) and the topography of the region varies from hilly escarpment in the western and southern edges with an altitude of 1000-1500 m.a.s.l to lowland plains that fall in the altitude of 0-100 m.a.s.l. Around 95 percent of the region has a flat landscape with altitude decreasing towards North eastern parts. More than $95 \%$ of the rural population is pure pastoralists, typically transhumant, although there are few parts of the people, which are sedentary in some pocket areas (Philpott et al., 2005).

The Region has a total number of 2,546,790 cattle, 2,541,920 sheep, 4,398,590 goats, 884,290 camels, 189,330 donkeys, 900 horses and 3,340 mules (CSA, 2004). The Afar pastoral communities depend on multispecies livestock production. However, camels, sheep, goats, cattle, and donkeys constitute the main productive assets of the local community. The primary feed sources for this large number of livestock are rangelands composed of indigenous species of grasses, shrubs and fodder trees. Most of these grass species however, are subjected to continuous threat of genetic erosion and extinction due to over grazing, rangeland degradation, undesirable plant encroachment (like Prosopis juliflora, Parthenium hysterophorus, Calotropis procera, Tribulis terrestris, Sida ovata, Cryptostegia grandiflora etc.) (APARI, 2005) and also Acacia nubica dominantly invaded the rangeland areas in Chifra district (Mohammed, 2009).

Overgrazing is the main antropic factors leading to the deterioration of the perennial plant cover. Its negative effect is excessive removal of the living parts of the high range value species, which may lead to their extinction. This factor is being more harmful when coupled with the climate aridity effect (drought). Most of the 
grazing areas of Afar region are exposed to overgrazing because of this the availability of animal feed decline both in quality and quantity, due to this, the community always moved from one area to the other areas inorder to search animal feed and water but through journey the pastoralists were faced different problems with the neighboring region community and also emaciated animals were always never come back to the original place because they will die on the way before reach into temporary settlement area, even after rainy season the grazing areas of the region covered by annual grasses which stayed only for two to three months after that the vegetation become diminished. As result of this, productivity of the livestock is very low there by affecting the livelihood of the people.

So implementation of different traditional and modern rangeland management system is crucial inorder to rehabilitate the degraded and encroached grazing areas of Afar region. Research and development in rangeland improvement schemes is therefore an important investment in order to reverse the negative impacts of different rangeland degradations caused by biotic and/or biotic factors. Furthermore, improvement impacts enhance livestock performances with higher values for local and export markets and enable the contribution of the sub sector in boosting income generation by pastoralists.

\section{Specific Objectives}

$\checkmark$ To determine whether there are differences in plant community response to restoration treatments as measured by species composition, vegetation cover and density across treatments

$\checkmark$ To know the species diversity of the above ground vegetation

$\checkmark$ To identify best restoration technique or techniques would be more appropriate for a given plant community

$\checkmark$ To create awareness within the community on how to restore degraded rangeland

\section{Materials and Methods}

\subsection{Description of the Study area}

The study was undertaken in Chifra district of zone one (Awsi Rasu) of the Afar Regional State. It is located south west of Semera on the main road of Mile to Woldiya, which is about $162 \mathrm{~km}$ from the regional capital city (Semera). Chifra is climatically characterized as arid and semi-arid agro-ecological area, where livestock production is the main occupation of the community. The average temperature of the area is about $29^{\circ} \mathrm{C}$, and the rainfall is bimodal with erratic distribution, with the long rainy season (Kerma) is between Mid-June to MidSeptember and the short rainy season (Sugum) that occurs between March and April. The average annual rainfall is recorded to be between 400 and $600 \mathrm{~mm}$ (APARDB, 2006).

\subsection{Experimental Procedure}

Each study site was visually evaluated and identified three plant communities dominated by annual grasses, perennial grasses, and mixed annual and perennial grasses using the protocols described in Johnson and Simon (1987). Predominately annual communities were described as areas containing less than $10 \%$ native perennial grass. Mixed communities was described as areas containing between 15 and $25 \%$ native perennial grass, whereas predominately perennial communities were described as area containing more than $25 \%$ native perennial grass.

Six treatments were established: (1) ripping, mulching and seeding (2) ripping, manuling and seeding (3) ripping, mulching, manuling and seeding (4) control. The treatments were arranged in a randomized complete block design with factorial and replicated three times in each plant community. Each treatment plot was $3 \mathrm{~m}$ by $6 \mathrm{~m}$, and depending on the treatment assigned to the plot, measurements for the vegetations were taken from those plots. Seeds of perennials, large tufted and palatable grass species were selected for reseeding purpose i.e., Andropogon canaliculatus, Tetrapogon cenchriformis, Panicum coloratum and Chryspogon plumolosus. The above grass species were mixed in order to sown in two plant communities which are annual communities and mixed communities. The reseeding was taken at a rate of $120 \mathrm{~g} / \mathrm{spp} / 100 \mathrm{~m}^{2}$. Samples of each seeds were taken to the laboratory to assess the purity percentage. Immediately following seeding, compressed pellets uneaten leaf mulch were broadcasted on the seeded plots at a rate of 1,953 kg per ha. 
Treatment Combination

\begin{tabular}{|l|l|l|}
\hline No & Plant community & Restoration methods \\
\hline 1 & Annual communities & Control \\
\cline { 3 - 3 } & & Ripping, Mulching and Seeding \\
\cline { 3 - 3 } & & Ripping, Manuling and Seeding \\
\cline { 3 - 3 } & & Ripping, Mulching, Manuling and Seeding \\
\hline 2 & Mixed communities & Control \\
\cline { 3 - 3 } & & Ripping, Mulching and Seeding \\
\cline { 3 - 3 } & & Ripping, Manuling and Seeding \\
\cline { 3 - 3 } & & Ripping, Mulching, Manuling and Seeding \\
\hline 3 & Perennial communities & Control \\
\cline { 3 - 3 } & & Ripping and Mulching \\
\cline { 3 - 3 } & & Ripping and Manuling \\
\cline { 3 - 3 } & & Ripping, Mulching and Manuling \\
\hline
\end{tabular}

\subsection{Materials used and data collected}

\subsubsection{Germination Scores}

The germination of grasses assessed by randomly placing three quadrants (visual within structures) by giving the scores of (3.5 - 4= poor germination; $3-3.5=$ fair germination; $2=$ good germination; $1=$ excellent germination) for each plot/structure. A score was considered germinated when the radicle length was $2 \mathrm{~mm}$ or above.

\subsubsection{Basal cover}

Cover assessed using quadrate $(0.5 \mathrm{~m} \times 0.5 \mathrm{~m})$ by randomly placing in the structures. An area of $0.25 \mathrm{~m}^{2}$ was selected for detailed assessment, and divided into halves. One of these was further divided into quarters, one of which divided into eighths. All grasses in the selected $0.25 \mathrm{~m} 2$ per plot was cut, transferred while kept together, and drawn in the eighth part to facilitate visual estimations of basal covers of living parts. The rating of basal cover was considered 'excellent' when the eighth was completely filled (12.5\%) or 'very poor' when the cover was less than 3\% (Baars et al. 1997). Then, each percentage multiplied by four to convert to out of $100 \%$.

\subsubsection{Herbaceous species composition}

The grass species were clustered into 3-groups based on the desirability following indigenous knowledge and ecological status supported by information from literature survey. The desirability rating was based on their long-term response to grazing and palatability. Ecological status, as used here, indicates species composition classified as decreasers, increasers and invaders or pioneers as defined in Tainton (1999). Accordingly, highly desirable species included species that are decreasers and perennials with a high palatability based upon the pastoralists perceptions. The intermediate desirable species are those that increase in abundance with moderate over-utilization, and perennials, which are average or high in terms of their palatability. The less desirable species include those species that increase in abundance with severe or extremely severe over-utilization of rangelands. This group includes perennial and annual species that are less palatable (Tainton, 1999).

\subsubsection{Dry matter determination}

The dry matter production was taken by using quadrant $(0.5 \mathrm{~m} \mathrm{X} 0.5 \mathrm{~m})$ from each plot/structures. Four quadrants were taken from each sample plot and the herbaceous species were divided into grasses and non-grasses by hand separation. The grass species were sorted by species while the non-grass herbaceous was combined as forbs. The samples were oven-dried at $105^{\circ} \mathrm{C}$ for 24 hours and weighed in order to determine the dry matter content.

\subsection{Statistical Analysis and Interpretation}

The data obtained from the vegetation was subjected to ANOVA using the GLM procedure of Statistical Analytical System (SAS) (2001) computer software. A significant difference was detected through ANOVA with $\mathrm{P} \leq 0.05$ and Means were separated by Duncan's Multiple Range Test (DMRT).

\section{Result \& Discussion}

\subsection{Herbaceous Species Composition, Use Values \& Relative Abundance \\ 4.1.1 First Implementation Year Annual Plant Community}

In first year trial, total grass species recorded in Annual plant community was 12 (6 annuals and 6 perennials) but the frequency or the occurrence of annuals grasses greater than perennial grasses in each plots. In restoration techniques $(\mathrm{R}+\mathrm{M}+\mathrm{S} \& \mathrm{R}+\mathrm{Ma}+\mathrm{S})$, less desirable grass specie like Aristida adoenesis were commonly found and also undesirable grass species like Tragus beteronianus \& Brachiaria eruciformis were dominantly found in each experimental plots while in restoration techniques $(\mathrm{R}+\mathrm{M}+\mathrm{Ma}+\mathrm{S})$, desirable grass like Dactyloctenium 
aegypticum \& Digitaria milanjana were presented in each experimental plots.

\section{Mixed Plant Community}

In first year trial, total grass species recorded in Annual plant community was 11 (4 annuals and 7 perennials) but the frequency or the occurrence of annuals grasses greater than perennial grasses in each plots.

\section{Perennial Plant Community}

In first year trial, total grass species recorded in Annual plant community was 10 (2 annuals and 8 perennials) but the frequency or the occurrence of annuals grasses greater than perennial grasses in each plots.

Table 1:- Herbaceous Species Composition, Use Values and Relative Abundance in Different Plant Communities in the $1^{\text {st }}$ Implementation Year

\begin{tabular}{|c|c|c|c|c|c|c|c|c|c|c|}
\hline \multirow[t]{3}{*}{ Species Names } & \multirow[t]{3}{*}{$\mathrm{Cg}$} & \multicolumn{9}{|c|}{ Year I } \\
\hline & & \multicolumn{3}{|c|}{ Annual } & \multicolumn{3}{|c|}{ Mixed } & \multicolumn{3}{|c|}{ Perennial } \\
\hline & & $\mathbf{R i}+\mathbf{M u}+\mathbf{S e}$ & $\begin{array}{l}\mathrm{Ri}+ \\
\mathrm{Ma}+ \\
\mathbf{S e}\end{array}$ & $\begin{array}{l}\mathrm{Ri}+ \\
\mathrm{Mu}+ \\
\mathrm{Ma}+ \\
\mathrm{Se}\end{array}$ & $\mathrm{Ri}+\mathrm{Mu}+\mathbf{S e}$ & $\begin{array}{l}\mathrm{Ri}+ \\
\mathrm{Ma}+ \\
\mathrm{Se}\end{array}$ & $\begin{array}{l}\mathrm{Ri}+ \\
\mathrm{Mu}+ \\
\mathrm{Ma}+ \\
\mathrm{Se}\end{array}$ & $\begin{array}{l}\mathrm{Ri}+ \\
\mathrm{Mu}\end{array}$ & $\begin{array}{l}\mathrm{Ri}+ \\
\mathrm{Ma}\end{array}$ & $\begin{array}{l}\mathbf{R i}+ \\
\mathrm{Mu}+ \\
\mathbf{M a}\end{array}$ \\
\hline Eleusine multifolia & LD & $\mathrm{C}$ & $\mathrm{C}$ & $\mathrm{P}$ & $\mathrm{P}$ & $\mathrm{P}$ & $\mathrm{P}$ & - & - & - \\
\hline $\begin{array}{l}\text { Dactyloctenium } \\
\text { aegypticum }\end{array}$ & $\mathrm{D}$ & $P$ & $\mathrm{P}$ & $P$ & $\mathrm{P}$ & $\mathrm{C}$ & $\mathrm{C}$ & $\mathrm{C}$ & $\mathrm{C}$ & $\mathrm{C}$ \\
\hline $\begin{array}{l}\text { Eragrostis } \\
\text { teniufolia }\end{array}$ & LD & $\mathrm{D}$ & $\mathrm{D}$ & $P$ & - & - & - & - & - & - \\
\hline Panicum coloratum & HD & $\mathrm{P}$ & $\mathrm{P}$ & $\mathrm{C}$ & $\mathrm{C}$ & $\mathrm{C}$ & $\mathrm{C}$ & $\mathrm{C}$ & $\mathrm{C}$ & $\mathrm{C}$ \\
\hline $\begin{array}{l}\text { Chrysopogon } \\
\text { plumolosus }\end{array}$ & $\mathrm{D}$ & $P$ & $\mathrm{P}$ & $\mathrm{C}$ & $\mathrm{C}$ & $\mathrm{C}$ & $\mathrm{C}$ & $\mathrm{C}$ & $\mathrm{C}$ & $\mathrm{C}$ \\
\hline Aristida adoenesis & LD & $\mathrm{C}$ & $\mathrm{C}$ & $\mathrm{C}$ & - & - & - & $\mathrm{P}$ & $\mathrm{P}$ & $\mathrm{P}$ \\
\hline Cynodon dactylon & HD & - & - & - & $\mathrm{P}$ & $\mathrm{P}$ & $\mathrm{C}$ & $\mathrm{P}$ & $\mathrm{C}$ & $\mathrm{C}$ \\
\hline $\begin{array}{l}\text { Tragus } \\
\text { beteronianus }\end{array}$ & UD & $\mathrm{D}$ & $\mathrm{D}$ & $\mathrm{C}$ & $\mathrm{P}$ & $\mathrm{P}$ & $\mathrm{P}$ & - & - & - \\
\hline Digitaria milanjana & $\mathrm{D}$ & $\mathrm{P}$ & $\mathrm{P}$ & $\mathrm{P}$ & $\mathrm{P}$ & $\mathrm{P}$ & $\mathrm{C}$ & $\mathrm{P}$ & $\mathrm{C}$ & $\mathrm{C}$ \\
\hline $\begin{array}{l}\text { Andropogon } \\
\text { canaliculatus }\end{array}$ & $\mathrm{D}$ & $\mathrm{P}$ & $\mathrm{P}$ & $\mathrm{C}$ & $\mathrm{C}$ & $\mathrm{C}$ & $\mathrm{D}$ & $\mathrm{C}$ & $\mathrm{C}$ & $\mathrm{C}$ \\
\hline $\begin{array}{l}\text { Tetrapogon } \\
\text { cenchriformis }\end{array}$ & $\mathrm{D}$ & $\mathrm{P}$ & $\mathrm{P}$ & $\mathrm{C}$ & $\mathrm{C}$ & $\mathrm{C}$ & $\mathrm{D}$ & $\mathrm{C}$ & $\mathrm{C}$ & $\mathrm{C}$ \\
\hline $\begin{array}{l}\text { Brachiaria } \\
\text { eruciformis }\end{array}$ & UD & $\mathrm{C}$ & $\mathrm{C}$ & $\mathrm{P}$ & $P$ & $P$ & - & - & - & - \\
\hline Chloris prieurii & LD & $\mathrm{C}$ & $\mathrm{P}$ & $\mathrm{P}$ & $\mathrm{P}$ & $\mathrm{P}$ & $\mathrm{p}$ & $\mathrm{P}$ & $\mathrm{P}$ & $\mathrm{P}$ \\
\hline Cenchrus ciliaris & HD & - & - & - & - & - & - & $\mathrm{P}$ & $\mathrm{P}$ & $\mathrm{P}$ \\
\hline
\end{tabular}

Remark: $\mathrm{Cg}=$ Categories; $\mathrm{HD}=$ highly desirable; $\mathrm{D}=$ Desirable; $\mathrm{LD}=$ Less desirable; UD = Undesirable; $\mathrm{D}=$ Dominant $(>20 \%) ; \mathrm{C}=$ Common $(10-20 \%) ; \mathrm{P}=$ Present $(<10 \%$ of the total herbaceous plant $)$ and $-=$ absent

\subsubsection{Second Implementation Year}

\section{Annual Plant Community}

In second year trial, total grass species recorded in Annual plant community was 12 (5 annuals and 7 perennials) but the frequency or the occurrence of annuals grasses grater than perennial grasses in each plots. In restoration techniques $(\mathrm{R}+\mathrm{M}+\mathrm{S} \& \mathrm{R}+\mathrm{Ma}+\mathrm{S})$, less desirable grass species like Eleusine multifolia \& Aristida adoenesis were commonly found and also undesirable grass specie like Brachiaria eruciformis were commonly found in each experimental plots while in restoration techniques $(\mathrm{R}+\mathrm{M}+\mathrm{Ma}+\mathrm{S})$, desirable grass like Digitaria milanjana were present ed in each experimental plots.

\section{Mixed Plant Community}

In second year trial, total grass species recorded in Annual plant community was 13 (3 annuals and 10 perennials) but the frequency or the occurrence of annuals grasses grater than perennial grasses in each plots.

\section{Perennial Plant Community}

In second year trial, total grass species recorded in Annual plant community was 12 (2 annuals and 10 perennials). 
Table 2:- Herbaceous Species Composition, Use Values and Relative Abundance in Different Plant Communities in the $2^{\text {nd }}$ Implementation Year

\begin{tabular}{|c|c|c|c|c|c|c|c|c|c|c|}
\hline \multirow[t]{3}{*}{ Species Names } & \multirow[t]{3}{*}{$\mathrm{Cg}$} & \multicolumn{9}{|c|}{ Year II } \\
\hline & & \multicolumn{3}{|c|}{ Annual } & \multicolumn{3}{|c|}{ Mixed } & \multicolumn{3}{|c|}{ Perennial } \\
\hline & & $\mathbf{R i}+\mathbf{M u}+\mathbf{S e}$ & $\begin{array}{l}\mathrm{Ri}+ \\
\mathrm{Ma}+ \\
\mathrm{Se}\end{array}$ & $\begin{array}{l}\mathrm{Ri}+ \\
\mathrm{Mu}+ \\
\mathrm{Ma}+ \\
\mathrm{Se}\end{array}$ & $\mathbf{R i}+\mathbf{M u}+\mathbf{S e}$ & $\begin{array}{l}\mathrm{Ri}+ \\
\mathrm{Ma}+ \\
\mathrm{Se}\end{array}$ & $\begin{array}{l}\mathrm{Ri}+ \\
\mathrm{Mu}+ \\
\mathrm{Ma}+ \\
\mathrm{Se}\end{array}$ & $\begin{array}{l}\mathbf{R i}+ \\
\mathbf{M u}\end{array}$ & $\begin{array}{l}\mathbf{R i}+ \\
\text { Ma }\end{array}$ & $\begin{array}{l}\mathrm{Ri}+ \\
\mathrm{Mu}+ \\
\mathbf{M a}\end{array}$ \\
\hline $\begin{array}{l}\text { Bothriochloa } \\
\text { insculpta }\end{array}$ & $\mathrm{D}$ & - & - & - & $\mathrm{P}$ & $P$ & $P$ & $P$ & $\mathrm{P}$ & $\mathrm{P}$ \\
\hline $\begin{array}{l}\text { Dactyloctenium } \\
\text { aegypticum }\end{array}$ & $\mathrm{D}$ & $\mathrm{P}$ & $\mathrm{P}$ & $\mathrm{P}$ & $\mathrm{P}$ & $\mathrm{C}$ & $\mathrm{C}$ & $\mathrm{C}$ & $\mathrm{C}$ & $\mathrm{C}$ \\
\hline Eragrostis teniufolia & LD & $\mathrm{D}$ & $\mathrm{D}$ & $\mathrm{P}$ & - & - & - & - & - & - \\
\hline Panicum coloratum & HD & $\mathrm{P}$ & $\mathrm{P}$ & $\mathrm{C}$ & $\mathrm{C}$ & $\mathrm{C}$ & $\mathrm{D}$ & $\mathrm{D}$ & $\mathrm{D}$ & $\mathrm{C}$ \\
\hline $\begin{array}{l}\text { Chrysopogon } \\
\text { plumolosus }\end{array}$ & $\mathrm{D}$ & $\mathrm{P}$ & $\mathrm{P}$ & $\mathrm{C}$ & $\mathrm{C}$ & $\mathrm{C}$ & $\mathrm{D}$ & $\mathrm{C}$ & $\mathrm{D}$ & $\mathrm{C}$ \\
\hline Aristida adoenesis & LD & $\mathrm{C}$ & $\mathrm{C}$ & $\mathrm{C}$ & - & - & - & $P$ & $\mathrm{P}$ & $\mathrm{P}$ \\
\hline Cynodon dactylon & $\mathrm{HD}$ & - & - & - & $\mathrm{P}$ & $\mathrm{P}$ & $\mathrm{C}$ & $\mathrm{P}$ & $\mathrm{C}$ & $\mathrm{C}$ \\
\hline Tragus beteronianus & UD & $\mathrm{D}$ & $\mathrm{D}$ & $\mathrm{C}$ & $\mathrm{P}$ & $\mathrm{P}$ & $\mathrm{P}$ & - & - & - \\
\hline Digitaria milanjana & $\mathrm{D}$ & $\mathrm{P}$ & $\mathrm{P}$ & $\mathrm{P}$ & $\mathrm{P}$ & $\mathrm{P}$ & $\mathrm{C}$ & $\mathrm{P}$ & $\mathrm{C}$ & $\mathrm{C}$ \\
\hline $\begin{array}{l}\text { Andropogon } \\
\text { canaliculatus }\end{array}$ & $\mathrm{D}$ & $\mathrm{P}$ & $\mathrm{P}$ & $\mathrm{C}$ & $\mathrm{C}$ & $\mathrm{C}$ & $\mathrm{D}$ & $\mathrm{C}$ & $\mathrm{C}$ & $\mathrm{C}$ \\
\hline $\begin{array}{l}\text { Tetrapogon } \\
\text { cenchriformis }\end{array}$ & $\mathrm{D}$ & $\mathrm{P}$ & $\mathrm{P}$ & $\mathrm{C}$ & $\mathrm{C}$ & $\mathrm{C}$ & $\mathrm{D}$ & $\mathrm{C}$ & $\mathrm{C}$ & $\mathrm{C}$ \\
\hline $\begin{array}{l}\text { Brachiaria } \\
\text { eruciformis }\end{array}$ & UD & $\mathrm{C}$ & $\mathrm{C}$ & $\mathrm{P}$ & $\mathrm{P}$ & $P$ & - & - & - & - \\
\hline $\begin{array}{l}\text { Cenchrus } \\
\text { pennisetiformis }\end{array}$ & HD & - & - & - & $\mathrm{p}$ & $\mathrm{p}$ & $\mathrm{p}$ & $\mathrm{P}$ & $\mathrm{P}$ & $\mathrm{P}$ \\
\hline Cenchrus ciliaris & $\mathrm{HD}$ & $\mathrm{C}$ & $\mathrm{P}$ & $\mathrm{P}$ & $\mathrm{P}$ & $\mathrm{P}$ & $\mathrm{p}$ & $\mathrm{P}$ & $\mathrm{P}$ & $\mathrm{P}$ \\
\hline Chloris prieurii & LD & - & - & - & - & - & - & $\mathrm{P}$ & $\mathrm{P}$ & $\mathrm{P}$ \\
\hline Eleusine multifolia & LD & $\mathrm{C}$ & $\mathrm{C}$ & $\mathrm{P}$ & $\mathrm{P}$ & $\mathrm{P}$ & $\mathrm{P}$ & - & - & - \\
\hline
\end{tabular}

Remark: $\mathrm{Cg}=$ Categories; $\mathrm{HD}=$ Highly desirable; $\mathrm{D}=$ Desirable; $\mathrm{LD}=$ Less desirable; $\mathrm{UD}=\mathrm{Undesirable} ; \mathrm{D}=$ Dominant $(>20 \%) ; \mathrm{C}=$ Common $(10-20 \%) ; \mathrm{P}=$ Present $(<10 \%$ of the total herbaceous plant $)$ and $-=$ Absent

\subsubsection{Third Implementation Year}

\section{Annual Plant Community}

In third year trial, total grass species recorded in Annual plant community was 13 (5 annuals and 8 perennials) but the frequency or the occurrence of annuals grasses greater than perennial grasses in each plots.

\section{Mixed Plant Community}

In third year trial, total grass species recorded in Annual plant community was 13 (3 annuals and 10 perennials) but the frequency or the occurrence of annuals grasses greater than perennial grasses in each plots.

\section{Perennial Plant Community}

In third year trial, total grass species recorded in Annual plant community was 14 (3 annuals and 11 perennials).

Table 3:- Herbaceous Species Composition, Use Values and Relative Abundance in Different Plant Communities in the $3^{\text {rd }}$ Implementation Year

\begin{tabular}{|c|c|c|c|c|c|c|c|c|c|c|}
\hline \multirow[t]{3}{*}{ Species Names } & \multirow[t]{3}{*}{$\mathrm{Cg}$} & \multicolumn{9}{|c|}{ Year III } \\
\hline & & \multicolumn{3}{|c|}{ Annual } & \multicolumn{3}{|c|}{ Mixed } & \multicolumn{3}{|c|}{ Perennial } \\
\hline & & $\mathbf{R i}+\mathbf{M u}+\mathbf{S e}$ & $\begin{array}{l}\mathrm{Ri}+ \\
\mathrm{Ma}+ \\
\mathrm{Se}\end{array}$ & $\begin{array}{l}\mathrm{Ri}+ \\
\mathrm{Mu}+ \\
\mathrm{Ma}+ \\
\mathrm{Se}\end{array}$ & $\mathbf{R i}+\mathbf{M u}+\mathbf{S e}$ & $\begin{array}{l}\mathrm{Ri}+ \\
\mathrm{Ma}+ \\
\mathrm{Se}\end{array}$ & $\begin{array}{l}\mathrm{Ri}+ \\
\mathrm{Mu}+ \\
\mathrm{Ma}+ \\
\mathrm{Se}\end{array}$ & $\begin{array}{l}\mathrm{Ri}+ \\
\mathrm{Mu}\end{array}$ & $\begin{array}{l}\mathrm{Ri}+ \\
\mathrm{Ma}\end{array}$ & $\begin{array}{l}\mathbf{R i}+ \\
\mathbf{M u}+ \\
\mathbf{M a}\end{array}$ \\
\hline $\begin{array}{l}\text { Bothriochloa } \\
\text { insculpta }\end{array}$ & $\mathrm{D}$ & - & - & - & $\mathrm{P}$ & $\mathrm{P}$ & $\mathrm{P}$ & $\mathrm{P}$ & $\mathrm{P}$ & $\mathrm{P}$ \\
\hline $\begin{array}{l}\text { Dactyloctenium } \\
\text { aegypticum }\end{array}$ & $\mathrm{D}$ & $\mathrm{P}$ & $P$ & $\mathrm{P}$ & $\mathrm{P}$ & $\mathrm{C}$ & $\mathrm{C}$ & $\mathrm{C}$ & $\mathrm{C}$ & $\mathrm{C}$ \\
\hline Eragrostis teniufolia & LD & $\mathrm{D}$ & $\mathrm{D}$ & $\mathrm{P}$ & - & - & - & - & - & - \\
\hline Panicum coloratum & HD & $\mathrm{P}$ & $P$ & $\mathrm{C}$ & $\mathrm{C}$ & $\mathrm{C}$ & $\mathrm{D}$ & $\mathrm{D}$ & $\mathrm{D}$ & $\mathrm{C}$ \\
\hline $\begin{array}{l}\text { Chrysopogon } \\
\text { plumolosus }\end{array}$ & $\mathrm{D}$ & $P$ & $P$ & $\mathrm{C}$ & $\mathrm{C}$ & $\mathrm{C}$ & $\mathrm{D}$ & $\mathrm{C}$ & $\mathrm{D}$ & $\mathrm{C}$ \\
\hline
\end{tabular}




\begin{tabular}{|c|c|c|c|c|c|c|c|c|c|c|}
\hline \multirow[t]{3}{*}{ Species Names } & \multirow[t]{3}{*}{$\mathrm{Cg}$} & \multicolumn{9}{|c|}{ Year III } \\
\hline & & \multicolumn{3}{|c|}{ Annual } & \multicolumn{3}{|c|}{ Mixed } & \multicolumn{3}{|c|}{ Perennial } \\
\hline & & $\mathbf{R i}+\mathbf{M u}+\mathbf{S e}$ & $\begin{array}{l}\mathrm{Ri}+ \\
\mathrm{Ma}+ \\
\mathrm{Se}\end{array}$ & $\begin{array}{l}\mathrm{Ri}+ \\
\mathrm{Mu}+ \\
\mathrm{Ma}+ \\
\mathrm{Se}\end{array}$ & $\mathbf{R i}+\mathbf{M u}+\mathbf{S e}$ & $\begin{array}{l}\mathbf{R i}+ \\
\mathrm{Ma}+ \\
\mathbf{S e}\end{array}$ & $\begin{array}{l}\mathrm{Ri}+ \\
\mathrm{Mu}+ \\
\mathrm{Ma}+ \\
\mathrm{Se}\end{array}$ & $\begin{array}{l}\mathrm{Ri}+ \\
\mathrm{Mu}\end{array}$ & $\begin{array}{l}\mathbf{R i}+ \\
\text { Ma }\end{array}$ & $\begin{array}{l}\mathrm{Ri}^{+} \\
\mathrm{Mu}+ \\
\mathrm{Ma}\end{array}$ \\
\hline Aristida adoenesis & LD & $\mathrm{C}$ & $\mathrm{C}$ & $\mathrm{C}$ & - & - & - & $\mathrm{P}$ & $\mathrm{P}$ & $\mathrm{P}$ \\
\hline Cynodon dactylon & $\mathrm{HD}$ & - & - & - & $\mathrm{P}$ & $\mathrm{P}$ & $\mathrm{C}$ & $\mathrm{P}$ & $\mathrm{C}$ & $\mathrm{C}$ \\
\hline Tragus beteronianus & UD & $\mathrm{D}$ & $\mathrm{D}$ & $\mathrm{C}$ & $\mathrm{P}$ & $\mathrm{P}$ & $\mathrm{P}$ & - & - & - \\
\hline Digitaria milanjana & $\mathrm{D}$ & $\mathrm{P}$ & $\mathrm{P}$ & $\mathrm{P}$ & $\mathrm{P}$ & $\mathrm{P}$ & $\mathrm{C}$ & $\mathrm{P}$ & $\mathrm{C}$ & $\mathrm{C}$ \\
\hline $\begin{array}{l}\text { Andropogon } \\
\text { canaliculatus }\end{array}$ & $\mathrm{D}$ & $P$ & $\mathrm{P}$ & $\mathrm{C}$ & $\mathrm{C}$ & $\mathrm{C}$ & $\mathrm{D}$ & $\mathrm{C}$ & $\mathrm{C}$ & $\mathrm{C}$ \\
\hline $\begin{array}{l}\text { Tetrapogon } \\
\text { cenchriformis }\end{array}$ & $\mathrm{D}$ & $\mathrm{P}$ & $\mathrm{P}$ & $\mathrm{C}$ & $\mathrm{C}$ & $\mathrm{C}$ & $\mathrm{D}$ & $\mathrm{C}$ & $\mathrm{C}$ & $\mathrm{C}$ \\
\hline $\begin{array}{l}\text { Brachiaria } \\
\text { eruciformis }\end{array}$ & UD & $\mathrm{C}$ & $\mathrm{C}$ & $\mathrm{P}$ & $\mathrm{P}$ & $\mathrm{P}$ & - & - & - & - \\
\hline $\begin{array}{l}\text { Cenchrus } \\
\text { pennisetiformis }\end{array}$ & HD & - & - & - & $\mathrm{p}$ & $\mathrm{p}$ & $\mathrm{p}$ & $\mathrm{P}$ & $\mathrm{P}$ & $\mathrm{P}$ \\
\hline Cenchrus ciliaris & HD & $\mathrm{C}$ & $\mathrm{P}$ & $\mathrm{P}$ & $\mathrm{P}$ & $\mathrm{P}$ & $\mathrm{p}$ & $\mathrm{P}$ & $\mathrm{P}$ & $\mathrm{P}$ \\
\hline Chloris prieurii & LD & - & - & - & - & - & - & $\mathrm{P}$ & $\mathrm{P}$ & $\mathrm{P}$ \\
\hline Eleusine multifolia & LD & $\mathrm{C}$ & $\mathrm{C}$ & $\mathrm{P}$ & $\mathrm{P}$ & $\mathrm{P}$ & $\mathrm{P}$ & - & - & - \\
\hline $\begin{array}{l}\text { Cymbopogon } \\
\text { giganteus }\end{array}$ & HD & - & - & - & - & $\mathrm{P}$ & $\mathrm{P}$ & $\mathrm{P}$ & $\mathrm{P}$ & $\mathrm{P}$ \\
\hline Teterapogon vilosus & $\mathrm{D}$ & - & - & - & - & - & - & $\mathrm{P}$ & $\mathrm{P}$ & $\mathrm{P}$ \\
\hline
\end{tabular}

Remark: $\mathrm{Cg}=$ Categories; HD = Highly desirable; $\mathrm{D}=$ Desirable; $\mathrm{LD}=$ Less desirable; $\mathrm{UD}=\mathrm{Undesirable} ; \mathrm{D}=$ Dominant $(>20 \%) ; \mathrm{C}=$ Common $(10-20 \%) ; \mathrm{P}=$ Present $(<10 \%$ of the total herbaceous plant $)$ and $-=$ Absent

4.2 Effect of Restoration Methods in Different Plant Community on Germination (scores), Basal cover (\%), Species Composition \& Dry matter yield (ton/ha) during the first Implementation Year

Variations of Germination, basal cover, species composition and dry matter yield were observed among treatments during the first implementation year. High germination score was observed in two different treatments which were Annual and Mixed plant community with Restoration method of $\mathrm{Ri}+\mathrm{Ma}+\mathrm{Mu}+\mathrm{Se}$ significantly difference at $(\mathrm{P}<0.05)$ than the other treatments. In case of Basal cover, Perennial plant community with Restoration method of $\mathrm{Ri}+\mathrm{Ma}+\mathrm{Mu}+\mathrm{Se}$ was significantly difference at $(\mathrm{P}<0.01)$ than other treatments while in species composition, Annual plant community with Restoration method of $\mathrm{Ri}+\mathrm{Ma}+\mathrm{Mu}+\mathrm{Se}$ was significantly difference at $(\mathrm{P}<0.05)$ than other treatments. In case of biomass yield, Perennial plant community with Restoration method of $\mathrm{Ri}+\mathrm{Ma}+\mathrm{Mu}+\mathrm{Se}$ was significantly difference at $(\mathrm{P}<0.05)$ than other treatments.

Table 4: ANOVA (LSM and SE) of Rangeland Parameters in Different Plant Community in First

\begin{tabular}{|l|l|c|c|c|}
\hline \multirow{2}{*}{ Parameter } & \multicolumn{2}{|c|}{ TRT } & \multicolumn{2}{c|}{ Year I } \\
\cline { 2 - 4 } & & Annual & Mixed & Perennial \\
\hline \multirow{5}{*}{ Germination (score) } & Control & $3.72+0.023^{\mathrm{d}}$ & $3.53+0.02^{\mathrm{c}}$ & $3.72+0.024^{\mathrm{c}}$ \\
\cline { 2 - 5 } & $\mathrm{Ri}+\mathrm{Mu}+\mathrm{Se}$ & $3.36+0.02^{\mathrm{c}}$ & $2.99+0.023^{\mathrm{b}}$ & $3.61+0.2^{\mathrm{c}}$ \\
\cline { 2 - 5 } & $\mathrm{Ri}+\mathrm{Ma}+\mathrm{Se}$ & $3.11+0.02^{\mathrm{b}}$ & $2.79+0.024^{\mathrm{b}}$ & $3.32+0.0 .23^{\mathrm{b}}$ \\
\cline { 2 - 5 } & $\mathrm{Ri}+\mathrm{Ma}+\mathrm{Mu}+\mathrm{Se}$ & $2.83+0.023^{\mathrm{a}}$ & $2.22+0.02^{\mathrm{a}}$ & $3.12+0.2^{\mathrm{a}}$ \\
\hline \multirow{5}{*}{ Basal cover (\%) } & Control & $25.56+2.25^{\mathrm{d}}$ & $29.7+2.99^{\mathrm{c}}$ & $35.2+3.148^{\mathrm{c}}$ \\
\cline { 2 - 5 } & $\mathrm{Ri}+\mathrm{Mu}+\mathrm{Se}$ & $28.6+1.55^{\mathrm{c}}$ & $32.9+2.65^{\mathrm{c}}$ & $43.6+2.48^{\mathrm{b}}$ \\
\cline { 2 - 5 } & $\mathrm{Ri}+\mathrm{Ma}+\mathrm{Se}$ & $30.33+2.80^{\mathrm{b}}$ & $36.1+2.26^{\mathrm{b}}$ & $49.4+3.36^{\mathrm{a}}$ \\
\cline { 2 - 5 } & $\mathrm{Ri}+\mathrm{Ma}+\mathrm{Mu}+\mathrm{Se}$ & $31.26+2.71^{\mathrm{a}}$ & $42.2+3.05^{\mathrm{a}}$ & $51.0+4.08^{\mathrm{a}}$ \\
\hline \multirow{5}{*}{ Species composition } & $\mathrm{Control}$ & $8.0+2.0^{\mathrm{d}}$ & $6.33+1.52^{\mathrm{c}}$ & $7.0+2.0^{\mathrm{c}}$ \\
\cline { 2 - 5 } & $\mathrm{Ri}+\mathrm{Mu}+\mathrm{Se}$ & $12.0+2.0^{\mathrm{c}}$ & $7.33+2.08^{\mathrm{c}}$ & $8.33+2.51^{\mathrm{b}}$ \\
\cline { 2 - 5 } & $\mathrm{Ri}+\mathrm{Ma}+\mathrm{Se}$ & $13.0+2.0^{\mathrm{b}}$ & $10.0+2.0^{\mathrm{b}}$ & $8.66+1.52^{\mathrm{b}}$ \\
\cline { 2 - 5 } & $\mathrm{Ri}+\mathrm{Ma}+\mathrm{Mu}+\mathrm{Se}$ & $14.0+2.0^{\mathrm{a}}$ & $12.3+2.51^{\mathrm{a}}$ & $10.0+2.0^{\mathrm{a}}$ \\
\hline
\end{tabular}




\begin{tabular}{|l|l|c|c|c|}
\hline \multirow{2}{*}{ Parameter } & \multicolumn{2}{|c|}{ TRT } & \multicolumn{3}{c|}{ Year I } \\
\cline { 2 - 5 } & & Annual & Mixed & Perennial \\
\hline \multirow{3}{*}{ Biomass } & Control & $0.226+0.125^{\mathrm{d}}$ & $0.83+0.07^{\mathrm{c}}$ & $1.00+0.19^{\mathrm{d}}$ \\
\cline { 2 - 5 } & $\mathrm{Ri}+\mathrm{Mu}+\mathrm{Se}$ & $0.34+0.14^{\mathrm{c}}$ & $0.95+0.2^{\mathrm{b}}$ & $1.16+0.17^{\mathrm{c}}$ \\
\cline { 2 - 5 } & $\mathrm{Ri}+\mathrm{Ma}+\mathrm{Se}$ & $0.63+0.18^{\mathrm{b}}$ & $1.14+0.13^{\mathrm{a}}$ & $1.56+0.16^{\mathrm{b}}$ \\
\cline { 2 - 5 } & $\mathrm{Ri}+\mathrm{Ma}+\mathrm{Mu}+\mathrm{Se}$ & $0.83+0.18^{\mathrm{a}}$ & $1.16+0.16^{\mathrm{a}}$ & $1.79+0.14^{\mathrm{a}}$ \\
\hline
\end{tabular}

$\checkmark \quad{ }^{\mathrm{a}-\mathrm{d}}$ means with different superscripts letters along row differ significantly $(\mathrm{p}<0.05)$

$\checkmark \quad{ }^{*}$ Germination $(3.5-4=$ poor; 3-3.5= fair;2= good; $1=$ excellent)

4.3 Effect of Restoration Methods in Different Plant Community on Germination (scores), Basal cover (\%), Species Composition \& Dry matter yield (ton/ha) during the Second Implementation Year

Germination score, basal cover, species composition and dry matter yield were significantly difference among treatments during the second season. High germination score was observed Perennial plant community with Restoration method of $\mathrm{Ri}+\mathrm{Ma}+\mathrm{Mu}+\mathrm{Se}$ significantly difference at $(\mathrm{P}<0.05)$ than the other treatments. In Perennial plant community with Restoration method of $\mathrm{Ri}+\mathrm{Ma}+\mathrm{Mu}+\mathrm{Se}$, the basal cover and biomass yield were significantly difference at $(\mathrm{P}<0.05)$ than the remaining treatment combination. In case of species composition, Annual plant community with Restoration method of $\mathrm{Ri}+\mathrm{Ma}+\mathrm{Mu}+\mathrm{Se}$ was highest number compare to other treatments which was 14.66 .

Table 5: ANOVA (LSM and SE) of Rangeland Parameters in Different Plant Community in Second Implementation Year

\begin{tabular}{|l|l|c|c|c|}
\hline \multirow{2}{*}{ Parameter } & \multicolumn{2}{|c|}{ TRT } & \multicolumn{3}{|c|}{ Year II } \\
\cline { 2 - 5 } & & \multicolumn{1}{|c|}{ Annual } & Mixed & Perennial \\
\hline Germination (score) & Control & $3.59+0.79^{\mathrm{d}}$ & $3.41+0.12^{\mathrm{d}}$ & $3.2+0.1^{\mathrm{d}}$ \\
\cline { 2 - 5 } & $\mathrm{Ri}+\mathrm{Mu}+\mathrm{Se}$ & $3.126+0.11^{\mathrm{c}}$ & $2.91+0.06^{\mathrm{c}}$ & $2.79+0.75^{\mathrm{c}}$ \\
\cline { 2 - 5 } & $\mathrm{Ri}+\mathrm{Ma}+\mathrm{Se}$ & $2.90+0.083^{\mathrm{b}}$ & $2.64+0.09^{\mathrm{b}}$ & $2.32+0.04^{\mathrm{b}}$ \\
\cline { 2 - 5 } & $\mathrm{Ri}+\mathrm{Ma}+\mathrm{Mu}+\mathrm{Se}$ & $2.33+0.07^{\mathrm{a}}$ & $2.21+0.09^{\mathrm{a}}$ & $1.98+0.077^{\mathrm{a}}$ \\
\hline Basal cover (\%) & Control & $28.7+2.32^{\mathrm{d}}$ & $33.2+2.58^{\mathrm{d}}$ & $42.9+3.14^{\mathrm{c}}$ \\
\cline { 2 - 5 } & $\mathrm{Ri}+\mathrm{Mu}+\mathrm{Se}$ & $31.13+3.88^{\mathrm{c}}$ & $36.5+1.87^{\mathrm{c}}$ & $47.23+2.45^{\mathrm{b}}$ \\
\cline { 2 - 5 } & $\mathrm{Ri}+\mathrm{Ma}+\mathrm{Se}$ & $33.0+3.17^{\mathrm{b}}$ & $43.1+2.88^{\mathrm{b}}$ & $47.4+3.38^{\mathrm{b}}$ \\
\cline { 2 - 5 } & $\mathrm{Ri}+\mathrm{Ma}+\mathrm{Mu}+\mathrm{Se}$ & $34.9+2.20^{\mathrm{a}}$ & $47.8+2.15^{\mathrm{a}}$ & $53.0+2.4^{\mathrm{a}}$ \\
\hline Species composition & $\mathrm{Control}$ & $10.0+2.0^{\mathrm{d}}$ & $8.0+2.0^{\mathrm{d}}$ & $8.0+0.0^{\mathrm{c}}$ \\
\cline { 2 - 5 } & $\mathrm{Ri}+\mathrm{Mu}+\mathrm{Se}$ & $12.3+1.154^{\mathrm{c}}$ & $9.0+1.73^{\mathrm{c}}$ & $8.66+1.52^{\mathrm{c}}$ \\
\cline { 2 - 5 } & $\mathrm{Ri}+\mathrm{Ma}+\mathrm{Se}$ & $13.66+1.52^{\mathrm{b}}$ & $12.0+2.0^{\mathrm{b}}$ & $9.6+0.57^{\mathrm{b}}$ \\
\cline { 2 - 5 } & $\mathrm{Ri}+\mathrm{Ma}+\mathrm{Mu}+\mathrm{Se}$ & $14.66+1.52^{\mathrm{a}}$ & $13.0+2.0^{\mathrm{a}}$ & $11.0+1.0^{\mathrm{a}}$ \\
\hline \multirow{5}{*}{ Biomass } & $\mathrm{Control}$ & $0.86+0.13^{\mathrm{d}}$ & $1.15+0.16^{\mathrm{d}}$ & $1.05+0.19^{\mathrm{d}}$ \\
\hline & $\mathrm{Ri}+\mathrm{Mu}+\mathrm{Se}$ & $0.99+0.20^{\mathrm{c}}$ & $1.3+0.08^{\mathrm{c}}$ & $1.34+0.19^{\mathrm{c}}$ \\
\hline & $\mathrm{Ri}+\mathrm{Ma}+\mathrm{Se}$ & $1.36+0.06^{\mathrm{b}}$ & $1.55+0.17 \mathrm{~b}$ & $1.83+0.12^{\mathrm{b}}$ \\
\hline & $\mathrm{Ri}+\mathrm{Ma}+\mathrm{Mu}+\mathrm{Se}$ & $1.54+0.17^{\mathrm{a}}$ & $1.9+0.08^{\mathrm{a}}$ & $2.043+0.09^{\mathrm{a}}$ \\
\hline
\end{tabular}

$\checkmark \quad$ a-d means with different superscripts letters along row differ significantly $(\mathrm{p}<0.05)$

$\checkmark \quad$ *Germination (3.5-4= poor; 3-3.5= fair;2= good; $1=$ excellent)

4.6 Effect of Restoration Methods in Different Plant Community on Germination (scores), Basal cover (\%), Species Composition \& Dry matter yield (ton/ha) during the third Implementation Year

High germination score was observed in Mixed plant community with Restoration Methods of $\mathrm{Ri}+\mathrm{Ma}+\mathrm{Mu}+\mathrm{Se}$, significantly difference at $(\mathrm{P}<0.05)$ than the remaining treatment combination. In Basal cover $\&$ biomass yield, Perennial plant community with Restoration Methods of $\mathrm{Ri}+\mathrm{Ma}+\mathrm{Mu}+\mathrm{Se}$ was significantly difference at $(\mathrm{P}<0.05)$ than other treatment combination. In Perennial plant community with Restoration Methods of $\mathrm{Ri}+\mathrm{Ma}+\mathrm{Mu}+\mathrm{Se}$, the basal cover \& species composition were highest number compare to other treatments which were $74.1 \%$ \& 2.37 respectively. In case of species composition, Annual plant community with Restoration method of Ri $+\mathrm{Ma}+\mathrm{Mu}+\mathrm{Se}$ was highest number compare to other treatments which was 17.3. 
Table 6: ANOVA (LSM and SE) of Rangeland Parameters in Different Plant Community in Third Implementation Year

\begin{tabular}{|c|c|c|c|c|}
\hline \multirow[t]{2}{*}{ Parameter } & \multirow{2}{*}{ TRT } & \multicolumn{3}{|c|}{ Year III } \\
\hline & & Annual & Mixed & Perennial \\
\hline \multirow[t]{4}{*}{ Germination (score) } & Control & $3.72+0.1^{\mathrm{c}}$ & $3.55+0.176^{\mathrm{c}}$ & $3.46+0.27^{\mathrm{c}}$ \\
\hline & $\mathrm{Ri}+\mathrm{Mu}+\mathrm{Se}$ & $3.6+0.1^{\mathrm{c}}$ & $3.4+0.21^{\mathrm{c}}$ & $3.39+0.24^{\mathrm{c}}$ \\
\hline & $\mathrm{Ri}+\mathrm{Ma}+\mathrm{Se}$ & $3.33+1.377^{\mathrm{b}}$ & $2.91+0.77^{\mathrm{b}}$ & $3.2+0.304^{\mathrm{b}}$ \\
\hline & $\mathrm{Ri}+\mathrm{Ma}+\mathrm{Mu}+\mathrm{Se}$ & $3.15+0.13^{\mathrm{a}}$ & $1.33+0.17^{\mathrm{a}}$ & $2.97+0.25^{\mathrm{a}}$ \\
\hline \multirow[t]{4}{*}{ Basal cover $(\%)$} & Control & $31.0+2.21^{\mathrm{d}}$ & $36.1+3.31^{\mathrm{d}}$ & $48.33+5.95^{\mathrm{c}}$ \\
\hline & $\mathrm{Ri}+\mathrm{Mu}+\mathrm{Se}$ & $31.96+2.55^{\mathrm{c}}$ & $40.53+3.23^{\mathrm{c}}$ & $61.26+2.83^{b}$ \\
\hline & $\mathrm{Ri}+\mathrm{Ma}+\mathrm{Se}$ & $34.23+3.16^{\mathrm{b}}$ & $49.26+3.49^{b}$ & $65.93+4.10^{b}$ \\
\hline & $\mathrm{Ri}+\mathrm{Ma}+\mathrm{Mu}+\mathrm{Se}$ & $40.96+1.98^{\mathrm{a}}$ & $56.93+2.90^{\mathrm{a}}$ & $74.1+4.01^{\mathrm{a}}$ \\
\hline \multirow[t]{4}{*}{ Species composition } & Control & $11.66+1.52 \mathrm{c}$ & $9.33+0.57^{\mathrm{c}}$ & $8.66+0.57^{\mathrm{c}}$ \\
\hline & $\mathrm{Ri}+\mathrm{Mu}+\mathrm{Se}$ & $14.6+0.57 b$ & $13.6+0.57^{b}$ & $11.33+0.57^{\mathrm{b}}$ \\
\hline & $\mathrm{Ri}+\mathrm{Ma}+\mathrm{Se}$ & $15.0+1.73 b$ & $13.0+1.73^{\mathrm{b}}$ & $11.3+1.15^{\mathrm{b}}$ \\
\hline & $\mathrm{Ri}+\mathrm{Ma}+\mathrm{Mu}+\mathrm{Se}$ & $17.3+1.15 \mathrm{a}$ & $16.0+1.0^{\mathrm{a}}$ & $13.0+1.0^{\mathrm{a}}$ \\
\hline \multirow[t]{4}{*}{ Biomass } & Control & $1.26+0.47^{b}$ & $1.42+0.17^{\mathrm{c}}$ & $1.28+0.23^{\mathrm{d}}$ \\
\hline & $\mathrm{Ri}+\mathrm{Mu}+\mathrm{Se}$ & $1.13+0.09^{b}$ & $1.63+0.21^{b}$ & $1.6+0.16^{\mathrm{c}}$ \\
\hline & $\mathrm{Ri}+\mathrm{Ma}+\mathrm{Se}$ & $1.56+0.19^{\mathrm{a}}$ & $1.68+0.15^{b}$ & $2.2+0.13^{\mathrm{b}}$ \\
\hline & $\mathrm{Ri}+\mathrm{Ma}+\mathrm{Mu}+\mathrm{Se}$ & $1.81+0.16^{\mathrm{a}}$ & $1.75+0.39^{\mathrm{a}}$ & $2.37+0.11^{\mathrm{a}}$ \\
\hline
\end{tabular}

$\checkmark \quad$ a-d means with different superscripts letters along row differ significantly $(\mathrm{p}<0.05)$

$\checkmark \quad{ }^{*}$ Germination $(3.5-4=$ poor; 3-3.5= fair;2= good; $1=$ excellent)

4.7 Effect of Restoration methods in Different Plant Community on Biomass Production during the whole implementation years

In Perennial plant community with Restoration Methods of $\mathrm{Ri}+\mathrm{Ma}+\mathrm{Mu}+\mathrm{Se}$ was significant difference at $(\mathrm{P}<0.05)$ in the three consecutive seasons in Biomass production. The highest Biomass production was observed in the third season of implementation time which was 2.37 ton/ha while the lowest biomass production was observed in the first season of implementation period in control one which was 1 ton/ha. In Mixed plant community with Restoration Methods of $\mathrm{Ri}+\mathrm{Ma}+\mathrm{Mu}+\mathrm{Se}$ was significant difference at $(\mathrm{P}<0.05)$ in the three consecutive seasons in Biomass production. The highest Biomass production was observed in the third season of implementation time which was 1.75 ton/ha while the lowest biomass production was observed in the first season of implementation period in control one which was 0.83 ton/ha.

In Annual plant community with Restoration Methods of $\mathrm{Ri}+\mathrm{Ma}+\mathrm{Mu}+\mathrm{Se}$ was significant difference at $(\mathrm{P}<0.05)$ in the three consecutive seasons in Biomass production. The highest Biomass production was observed in the third season of implementation time which was 1.81 ton/ha while the lowest biomass production was observed in the first season of implementation period in control one which was $0.22 \mathrm{ton} / \mathrm{ha}$.

Table 7: ANOVA (LSM and SE) of Biomass production(ton/ha) in Different Restoration methods within the Consecutive Implementation Years

\begin{tabular}{|l|l|c|c|c|}
\hline \multicolumn{2}{|l|}{ Treatment Combinations } & \multicolumn{3}{c|}{ Biomass Production } \\
\cline { 3 - 5 } Annual & Control & Year 1 & Year 2 & Year 3 \\
\cline { 2 - 5 } & Ri+Mu+Se & $0.22+0.12^{\mathrm{c}}$ & $0.86+0.13^{\mathrm{b}}$ & $1.26+0.47^{\mathrm{a}}$ \\
\cline { 2 - 5 } & $\mathbf{R i}+\mathbf{M a}+\mathbf{S e}$ & $0.63+0.18^{\mathrm{c}}$ & $1.36+0.06^{\mathrm{b}}$ & $1.13+0.09^{\mathrm{a}}$ \\
\cline { 2 - 5 } & $\mathbf{R i}+\mathbf{M a}+\mathbf{M u}+\mathbf{S e}$ & $0.83+0.18^{\mathrm{c}}$ & $1.54+0.17^{\mathrm{b}}$ & $1.56+0.19^{\mathrm{a}}$ \\
\hline \multirow{4}{*}{ Mixed } & Control & $0.83+0.07^{\mathrm{c}}$ & $1.15+0.16^{\mathrm{b}}$ & $1.81+0.16^{\mathrm{a}}$ \\
\cline { 2 - 5 } & $\mathbf{R i}+\mathbf{M u}+\mathbf{S e}$ & $0.95+0.2^{\mathrm{c}}$ & $1.3+0.08^{\mathrm{b}}$ & $1.63+0.17^{\mathrm{a}}$ \\
\cline { 2 - 5 } & $\mathbf{R i}+\mathbf{M a}+\mathbf{S e}$ & $1.14+0.13^{\mathrm{b}}$ & $1.55+0.17^{\mathrm{a}}$ & $1.68+0.15^{\mathrm{a}}$ \\
\cline { 2 - 5 } & $\mathbf{R i}+\mathbf{M a}+\mathbf{M u}+\mathbf{S e}$ & $1.16+0.16^{\mathrm{c}}$ & $1.58+0.08^{\mathrm{b}}$ & $1.75+0.39^{\mathrm{a}}$ \\
\hline
\end{tabular}




\begin{tabular}{|c|c|c|c|c|}
\hline \multicolumn{2}{|c|}{ Treatment Combinations } & \multicolumn{3}{|c|}{ Biomass Production } \\
\hline & & Year 1 & Year 2 & Year 3 \\
\hline \multirow[t]{4}{*}{ Perennial } & Control & $1.00+0.19^{\mathrm{b}}$ & $1.05+0.19^{\mathrm{b}}$ & $1.28+0.23^{\mathrm{a}}$ \\
\hline & $\mathbf{R i}+\mathbf{M u}+\mathbf{S e}$ & $1.16+0.17^{\mathrm{c}}$ & $1.34+0.19^{\mathrm{b}}$ & $1.6+0.16^{\mathrm{a}}$ \\
\hline & $\mathbf{R i}+\mathbf{M a}+\mathbf{S e}$ & $1.56+0.16^{\mathrm{b}}$ & $1.83+0.12^{b}$ & $2.2+0.13^{\mathrm{a}}$ \\
\hline & $\mathbf{R i}+\mathrm{Ma}+\mathrm{Mu}+\mathrm{Se}$ & $1.79+0.14^{\mathrm{c}}$ & $2.043+0.09^{b}$ & $2.37+0.11^{\mathrm{a}}$ \\
\hline
\end{tabular}

$\checkmark \quad$ a-c means with different superscripts letters along column differ significantly $(\mathrm{p}<0.05)$

4.8 Effect of Restoration methods in Different Plant Community on Basal Cover during the whole implementation years

In Perennial plant community with Restoration Methods of $\mathrm{Ri}+\mathrm{Ma}+\mathrm{Mu}+\mathrm{Se}$ was significant difference at $(\mathrm{P}<0.05)$ in the three consecutive seasons in Basal cover. The highest percentage of basal cover was observed in the third season of implementation time which was 74.1 while the lowest percentage of basal cover was observed in the first season of implementation period which was 51.0. In Mixed plant community with Restoration Methods of $\mathrm{Ri}+\mathrm{Ma}+\mathrm{Mu}+\mathrm{Se}$ was significant difference at $(\mathrm{P}<0.05)$ in the three consecutive seasons in Basal cover. The highest percentage of basal cover was observed in the third season of implementation time which was 56.93 while the lowest percentage of basal cover was observed in the first season of implementation period which was 42.2.

In Annual plant community with Restoration Methods of $\mathrm{Ri}+\mathrm{Ma}+\mathrm{Mu}+\mathrm{Se}$ was significant difference at $(\mathrm{P}<0.05)$ in the three consecutive seasons in Basal cover. The highest percentage of basal cover was observed in the third season of implementation time which was 40.96 while the lowest biomass production was observed in the first season of implementation period which was 31.26.

Table 8: ANOVA (LSM and SE) of Basal Cover in Different Restoration methods within the Consecutive Implementation Years

\begin{tabular}{|c|c|c|c|c|}
\hline \multirow{2}{*}{\multicolumn{2}{|c|}{ Treatment Combinations }} & \multicolumn{3}{|c|}{ Basal Cover } \\
\hline & & Year 1 & Year 2 & Year 3 \\
\hline \multirow[t]{4}{*}{ Annual } & Control & $25.56+2.25^{\mathrm{c}}$ & $28.7+2.32^{b}$ & $31.0+2.21^{\mathrm{a}}$ \\
\hline & $\mathbf{R i}+\mathbf{M u}+\mathbf{S e}$ & $28.6+1.55^{b}$ & $31.13+3.88^{\mathrm{a}}$ & $31.96+2.55^{\mathrm{a}}$ \\
\hline & $\mathbf{R i}+\mathbf{M a}+\mathbf{S e}$ & $30.33+2.80^{b}$ & $33.0+3.17^{\mathrm{a}}$ & $34.23+3.16^{\mathrm{a}}$ \\
\hline & $\mathbf{R i}+\mathbf{M a}+\mathbf{M u}+\mathrm{Se}$ & $31.26+2.71^{\mathrm{c}}$ & $34.9+2.20^{\mathrm{b}}$ & $40.96+1.98^{\mathrm{a}}$ \\
\hline \multirow[t]{4}{*}{ Mixed } & Control & $29.7+2.99^{\mathrm{c}}$ & $33.2+2.58^{\mathrm{b}}$ & $36.1+3.31^{\mathrm{a}}$ \\
\hline & $\mathbf{R i}+\mathbf{M u}+\mathbf{S e}$ & $32.9+2.65^{\mathrm{c}}$ & $36.5+1.87^{\mathrm{b}}$ & $40.53+3.23^{\mathrm{a}}$ \\
\hline & $\mathbf{R i}+\mathbf{M a}+\mathbf{S e}$ & $36.1+2.26^{\mathrm{c}}$ & $43.1+2.88^{\mathrm{b}}$ & $49.26+3.49^{\mathrm{a}}$ \\
\hline & $\mathbf{R i}+\mathbf{M a}+\mathbf{M u}+\mathbf{S e}$ & $42.2+3.05^{\mathrm{c}}$ & $47.8+2.15^{\mathrm{b}}$ & $56.93+2.90^{\mathrm{a}}$ \\
\hline \multirow[t]{4}{*}{ Perennial } & Control & $35.2+3.148^{\mathrm{c}}$ & $42.9+3.14^{\mathrm{b}}$ & $48.33+5.95^{\mathrm{a}}$ \\
\hline & $\mathbf{R i}+\mathbf{M u}+\mathbf{S e}$ & $43.6+2.48^{\mathrm{b}}$ & $47.23+2.45^{\mathrm{b}}$ & $61.26+2.83^{\mathrm{a}}$ \\
\hline & $\mathbf{R i}+\mathbf{M a}+\mathbf{S e}$ & $49.4+3.36^{b}$ & $47.4+3.38^{\mathrm{b}}$ & $65.93+4.10^{\mathrm{a}}$ \\
\hline & $\mathbf{R i}+\mathbf{M a}+\mathbf{M u}+\mathbf{S e}$ & $51.0+4.08^{\mathrm{b}}$ & $53.0+2.4^{\mathrm{b}}$ & $74.1+4.01^{\mathrm{a}}$ \\
\hline
\end{tabular}

$\checkmark \quad$ a-c means with different superscripts letters along column differ significantly $(\mathrm{p}<0.05)$

\section{Conclusion \& Recommendation}

\subsection{Conclusion}

Generally from this finding we concluded that, implementation of different Restoration methods within three categories of plant communities has been improved range species composition, basal cover \& biomass production compared with the control one. In Annual plant community with Restoration Methods of $\mathrm{Ri}+$ $\mathrm{Ma}+\mathrm{Mu}+\mathrm{Se}$ showed good performance in biomass production than the other treatment combinations. The higher biomass production was harvested from the treatment combination of annual plant community with Restoration Methods of $\mathrm{Ri}+\mathrm{Ma}+\mathrm{Mu}+\mathrm{Se}$ in the $3 \mathrm{rd}$ season which was 1.81 tone/ha and the lowest result was the treatment combination of annual plant community with control one in the 1 st season which was 0.22 tone/ha. In Mixed plant community with Restoration Methods of $\mathrm{Ri}+\mathrm{Ma}+\mathrm{Mu}+\mathrm{Se}$ showed good performance in biomass production than the other treatment combinations. The higher biomass production was harvested from the 
treatment combination of mixed plant community with Restoration Methods of $\mathrm{Ri}+\mathrm{Ma}+\mathrm{Mu}+\mathrm{Se}$ in the 3 rd season which was 1.75 tone/ha and the lowest result was the treatment combination of mixed plant community with control one in the $1^{\text {st }}$ season which was 0.83 tone/ha

Therefore, we conclude that from this finding, perennial plant community with Restoration methods of $\mathrm{Ri}+\mathrm{Ma}+\mathrm{Mu}+\mathrm{Se}$ is more effective method to rehabilitate denuded rangeland areas in semi-arid areas and also Annual plant community with Restoration methods of $\mathrm{Ri}+\mathrm{Ma}+\mathrm{Mu}+\mathrm{Se}$ has shown dramatic result to improved range resources within short period of time. However, continuous onsite training \& practical demonstration was very crucial to enhance the adoption rate of those Restoration methods.

\subsection{Recommendation}

The following recommendations were derived from the data of the study and the observations during experimentation period

$\checkmark \quad$ Verification of the best suitable Restoration methods in order to promote at wide scale

$\checkmark$ Continues awareness rising to the community about the best suit Restoration methods

\section{References}

Afar Pastoral, Agricultural and Development Beuro (APADB), 2006. Baseline survey made on the Potential, Constraints, and Opportunity on the Production System of 29 woredas of Afar National Regional State.

Afar Pastoral and Agro-pastoral Research Institute (APARI), 2005. Rangeland management strategic plane Baars, R.M.T., E.C. Chileshe and D.R. Kalokoni, 1997. Technical notes: Range condition in high cattle density areas in the Western Province of Zambia. Tropical Grasslands, 31: 569573.

Central Statistical Authority (CSA), 2004. Ethiopian Statistical Abstract, Central Statistical Authority, Addis

Ababa Ethiopia.

Coppock, D. Layne, 1994. The Borena Plateau of Southern Ethiopia. Synthesis of Pastoral Research, Development and Change, 1980-91, ILCA, Addis Ababa, Ethiopia.

Friedel, M.H., W.A. Laycock and G.N. Basin, 2000. Assessing rangeland condition and trend. pp. 227-261. In: L.T. Mannetje and R.M. Jones (eds.). Filed Laboratory Methods for Grassland and Animal Production research. CABI, UK.

Mohammed, 2009. Assessment of Pastoral Perceptions, Range Condition and Chemical Composition of Major Feed Resources in Chifra District of Afar Regional State, Ethiopia

National Research council (NRC), 1990. The improvement of tropical and Sub-tropical rangelands. National Academy of Press, Washington Dc. USA 180p. 